\title{
Comprehensive economic evaluation of enhanced recovery after surgery in hepatectomy
}

Yihan Dong ${ }^{1,2}$, Yan Zhang ${ }^{1,2^{*}}$ (1) and Chengcheng Jin ${ }^{1,3}$

\begin{abstract}
Background: Enhanced recovery after surgery (ERAS) is attracting extensive attention and being widely applied to reduce postoperative stress and accelerate recovery. However, the economic benefits of ERAS are less clarified at the social level. We aimed to assess the economic impact of ERAS in hepatectomy from the perspectives of patients, hospitals and society, as well as identify the approach to create the economic benefits of ERAS.

Methods: By combining the literature and national statistical data, the cost-effectiveness framework was clarified, and parameter values were determined. Cost-effectiveness analysis, cost-benefit analysis and cost-minimisation analysis were used to compare ERAS and conventional treatment from the perspectives of patients, hospitals and society. The capital flow diagram was used to analyse the change between them.

Results: ERAS significantly reduced the economic burden of disease on patients ( $\$ 8935.02 \mathrm{vs} \$ 10,470.02)$. The hospital received an incremental benefit in ERAS (the incremental benefit cost ratio value is 1.09), and the total social cost was reduced ( $\$ 5958.67$ vs $\$ 6725.80$ ). Capital flow diagram analysis demonstrated that the average daily cost per capita in the ERAS group increased (\$669.51 vs $\$ 589.98$ ), whereas the benefits depended on the reduction of hospital stay and productivity loss.

Conclusion: The mechanism by which ERAS works is to reduce the average length of stay, thereby reducing the economic burden and productivity loss on patients and promoting the hospital bed turnover rate. Therefore, ERAS should further focus on accelerating the rehabilitation process, and more economic support (such as subsidies) should be given to hospitals to carry out ERAS.
\end{abstract}

Keywords: Enhanced recovery after surgery, Hepatectomy, Economic evaluation, Social benefit, Multiple perspective

\section{Background}

Enhanced recovery after surgery (ERAS) refers to the integrated measures centring on the entire disease cycle under the guidance of the multi-disciplinary team collaboration model, with the core goal of reducing the incidence of complications and accelerating postoperative

\footnotetext{
*Correspondence: yanzhang@hust.edu.cn

${ }^{1}$ School of Medicine and Health Management, Tongji Medical College, Huazhong University of Science and Technology, No.13 Hangkong Road, Qiaokou District, Wuhan 430030, Hubei, China

Full list of author information is available at the end of the article
}

rehabilitation [1]. Many studies have demonstrated the effectiveness and safety of ERAS, which reduces complication rates and length of hospital stay [2-4]. Therefore, ERAS has been widely used in research and practice in various clinical fields, including colorectal surgery, gastrointestinal surgery, urology, hepatobiliary surgery, orthopedics, and gynecology [5].

As an innovative clinical project, ERAS requires a large amount of equipment, capital and manpower. It should be preferentially cost-beneficial as well as socially beneficial to be favoured by financial hospital authorities. Published studies of ERAS economics indicates that ERAS

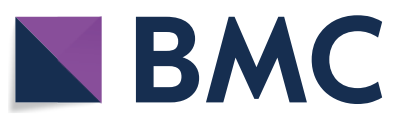

(c) The Author(s) 2021. Open Access This article is licensed under a Creative Commons Attribution 4.0 International License, which permits use, sharing, adaptation, distribution and reproduction in any medium or format, as long as you give appropriate credit to the original author(s) and the source, provide a link to the Creative Commons licence, and indicate if changes were made. The images or other third party material in this article are included in the article's Creative Commons licence, unless indicated otherwise in a credit line to the material. If material is not included in the article's Creative Commons licence and your intended use is not permitted by statutory regulation or exceeds the permitted use, you will need to obtain permission directly from the copyright holder. To view a copy of this licence, visit http://creativecommons.org/licenses/by/4.0/. The Creative Commons Public Domain Dedication waiver (http://creativeco mmons.org/publicdomain/zero/1.0/) applies to the data made available in this article, unless otherwise stated in a credit line to the data. 
has important economic value [6-9]. Domestic economic evaluation of ERAS for liver cancer, stomach cancer and colon cancer showed that the average total cost of ERAS group was lower than that of conventional group [7]. A meta-analysis of colorectal surgery found an average cost reduction of $\$ 3010$ with ERAS, and a meta-analysis of pancreatic surgery showed a $\$ 7020$ average cost reduction with ERAS [10]. However, most of these studies are based on a single perspective of patients, so ERAS has only been shown to reduce hospitalization costs for patients, while the economic benefits at the hospital and social level remain unclear. Moreover, incomplete cost components are involved. Costs are divided into direct costs, indirect costs and intangible costs. Since indirect costs and intangible costs are difficult to measure, they are not included in most studies.

In addition, due to the wide variety of ERAS protocols and their wide range of applications, there are some obstacles to the evaluation of economic benefits. According to expert consensus and guidelines, a series of ERAS items exists, which are unbalanced in terms of compliance and execution in practice [11-13]. Some so-called ERAS implement only a few items, which may lead to the abuse of ERAS. Studies have shown that strict implementation of standard ERAS pathways can lead to better health outcomes and economic benefits for patients [14]. As a result, ERAS protocols that do not meet standards are economically challenged, and some may even be economically ineffective. When evaluating the economic benefits of ERAS, it is also easy to make biased judgments that are higher or lower than the actual benefits, resulting in confusion in policy formulation and implementation.

Therefore, this study aims to assess the economic benefits of ERAS from the perspectives of patients, hospitals and society, as well as identify the approach to create economic and social benefits. Given the complexity of assessing the economic benefits of ERAS as a whole with different surgical procedures, hepatectomy was selected for our study. Hepatectomy (the ICD - 10 disease code is D18.013) is a widely used operation with heavy burden, which causes long-term stress reactions, high incidence of complications and slow postoperative recovery. It is also considered one of the most effective disease scenarios for ERAS $[15,16]$. Thus, we took hepatectomy as an example for our study.

\section{Methods}

\section{Study design}

This study intended to conduct an economic analysis of ERAS. The economic framework and cost composition of patients, hospitals and society were identified by literature review. This study included patients undergoing hepatectomy, who were divided into two groups, one group receiving ERAS care (ERAS group) and the other group receiving conventional surgery (control group). The costs and benefits of the two groups under different perspectives will be compared to obtain the economic value of ERAS. To avoid possible risks to the extrapolation rationality of the results due to the differences in social system and price, cost parameters in China were adopted in this study.

\section{Literature search}

A literature review was performed to construct the economic framework for ERAS. Searches were conducted in several databases, such as PubMed, EMBASE and Web of Science. Search terms included 'enhanced recovery after surgery', 'fast-track surgery,' 'ERAS, 'FTS', 'economic evaluation' and 'cost analysis'. Inclusion criteria: Economic evaluation of ERAS. Exclusion criteria: non-economic research, literature not available and using repeated data. After the retrieved literatures were de-duplicated, two researchers independently screened the titles and abstracts according to the literature inclusion and exclusion criteria, and then re-screened the full text. Then, the basic information of the included literature was extracted according to the pre-established data extraction form, including perspective, population, methods, indicators and results. Differences between the two researchers shall be resolved through discussion or consultation with a third party. The flow diagram of study selection is shown in Fig. 1.

\section{Model building and parameter determination}

Cost components and effect (benefit) items and the economic conceptual model were constructed in accordance with the cost framework proposed by Larg A et al [10, 17]. The components of cost from multiple perspectives are summarised in detail in Table 1.

This study estimated direct medical cost, direct nonmedical cost and productivity loss. Given that the method for measuring intangible cost was of low precision, it was excluded in the cost accounting scope. As for indirect cost, since the presence or specific number of family members as caregivers could not be determined from the literature, only the productivity loss due to the patient himself/herself was considered. The incidence of complications was selected as the effect indicator, and the hospital benefit integrated medical expenses charged and bed turnover rate.

The specific parameter value was determined based on the literature and national statistical data. Considering the regional differences in consumer price index (CPI) and the impact of time on currency, the currency values were converted to the 2018 national present value on the basis of the regional and national CPI levels and discount 


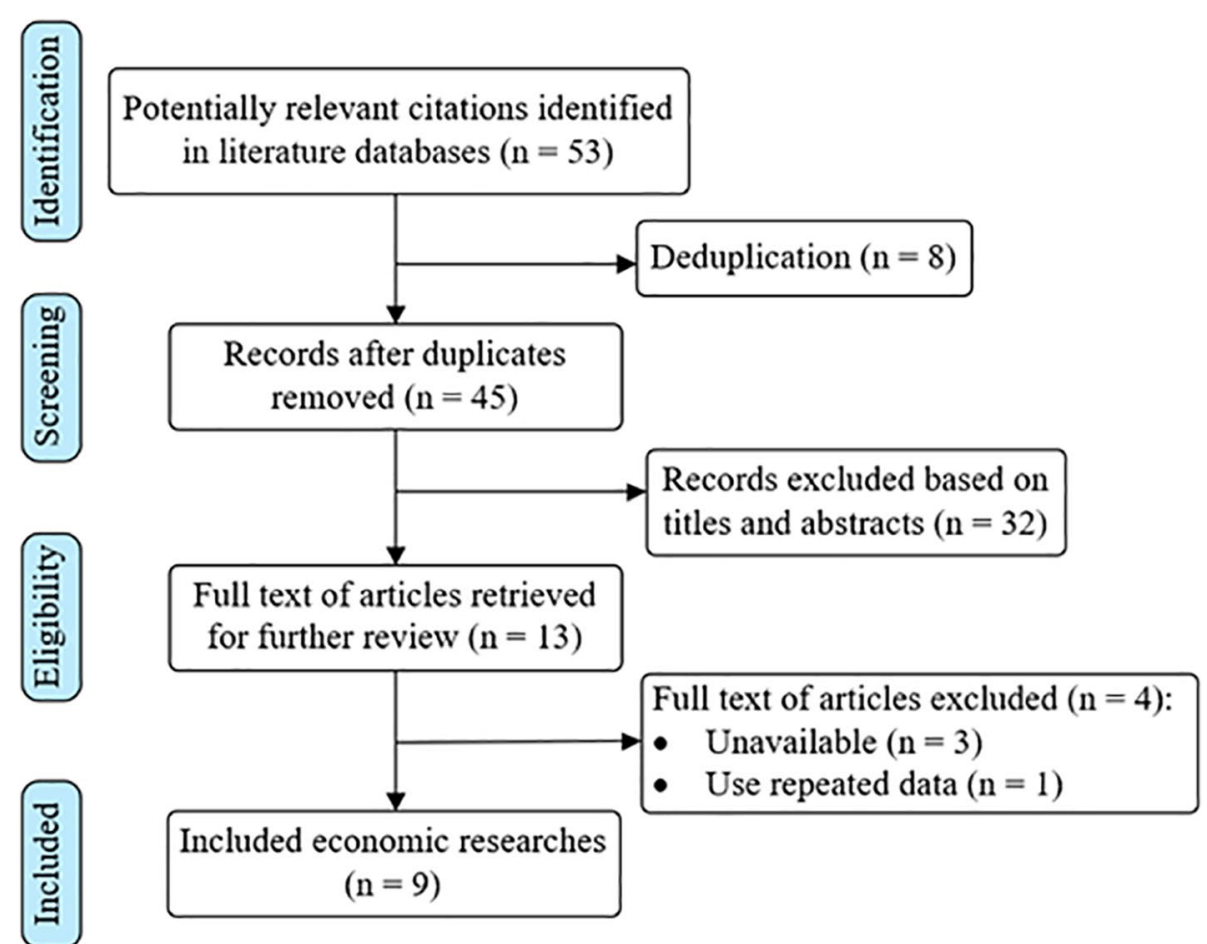

Fig. 1 Flow diagram of study selection

Table 1 Components of cost categorized by the type of cost from multiple perspectives

\begin{tabular}{|c|c|c|c|}
\hline Perspective & Direct cost & Indirect cost & Intangible cost \\
\hline Patients & $\begin{array}{l}\text { Direct medical cost: hospitalisation cost (medical expenses during pre-, } \\
\text { intra- and postoperative) } \\
\text { Direct non-medical cost: } \\
\text { food expenses; transportation; employment of care workers; others } \\
\text { (accommodation fees, etc) }\end{array}$ & $\begin{array}{l}\text { Productivity loss of } \\
\text { patients and their } \\
\text { families due to hospi- } \\
\text { talisation }\end{array}$ & $\begin{array}{l}\text { Pain and anxiety of patient and } \\
\text { their families caused by disease; } \\
\text { patient medical experience and } \\
\text { satisfaction }\end{array}$ \\
\hline Hospitals & $\begin{array}{l}\text { Standard input: } \\
\text { routine medical services; resource consumption; administrative manage- } \\
\text { ment } \\
\text { ERAS specific input: } \\
\text { salary of ERAS full-time nurse; quarterly ERAS meeting cost; ERAS database } \\
\text { COst; } \\
\text { ERAS patient log cost }\end{array}$ & - & $\begin{array}{l}\text { Labor and time cost of medical } \\
\text { staff }\end{array}$ \\
\hline Society & $\begin{array}{l}\text { Hospital standard input: } \\
\text { routine medical services; resource consumption; administrative manage- } \\
\text { ment } \\
\text { Hospital ERAS specific input: } \\
\text { salary of ERAS full-time nurse; quarterly ERAS meeting cost; ERAS database } \\
\text { cost; } \\
\text { ERAS patient log cost } \\
\text { Direct non-medical cost: } \\
\text { food expenses; transportation; employment of care workers; others } \\
\text { (accommodation fees, etc) }\end{array}$ & $\begin{array}{l}\text { Productivity loss of } \\
\text { patients and their } \\
\text { families due to hospi- } \\
\text { talisation }\end{array}$ & Psychosocial influence \\
\hline
\end{tabular}

rates for the current year [18-20]. All costs were primarily in Chinese Yuan Renminbi (CNY). The used exchange rate to US dollar (USD) was the one current on June 29, 2020: $1 \mathrm{CNY}=0.1413$ USD.

\section{Economic analysis methods and statistical analysis}

Cost-effectiveness analysis (CEA) was used from the patient perspective. CEA is to find a plan with the lowest cost and the best effect. The present analysis 
compared the cost and effectiveness of the ERAS and control groups.

Cost-benefit analysis (CBA) and univariate sensitivity analysis were conducted from the hospital perspective. CBA compares expected benefits and expected costs, using the incremental benefit cost ratio (IBCR) as the analysis index. IBCR represents the ratio of incremental benefit to incremental cost in ERAS and control groups. Univariate sensitivity analysis evaluates the influence of the changes of several major variables within a certain range on the results. Three variables were selected in this study: bed turnover rate, hospitalisation cost and hospital profit margin.

$$
\mathrm{IBCR}=(\mathrm{B} 1-\mathrm{B} 2) /(\mathrm{C} 1-\mathrm{C} 2)=\Delta \mathrm{B} / \Delta \mathrm{C}
$$

Cost-minimisation analysis (CMA) was conducted to evaluate the social resource consumption of ERAS. CMA calculated the total cost by adding all costs in ERAS group/control group and compared them.

Statistical analysis and comprehensive evaluation were performed using Excel 2016. The capital flow diagram was used to analyse changes in per capita economic burden, hospital income and total social cost. Per capita economic burden refers to the economic loss caused by disease, including direct and indirect economic loss. Hospital income refers to net revenue, which is equal to medical fees charged to patients minus hospital operating costs. Total social cost refers to the total consumption of social resources.

\section{Results}

\section{Summary of parameter values}

The specific parameter values are summarised in detail in Table 2.

\section{Results of cost-effectiveness analysis}

For patients, cost-effectiveness analysis showed that the cost of ERAS group (\$8935.02) was lower than that of the control group $(\$ 10,470.02)$, and the incidence of complications in ERAS group (6.33\%) was also lower than that of the control group (12.40\%) (Table 3). This suggests that ERAS has safety and economic advantages over conventional treatments.

\section{Results of cost-benefit analysis and univariate sensitivity analysis}

For hospitals, the cost and benefit of ERAS were higher than those of control group (Table 4). When the IBCR value is greater than or equal to 1 , ERAS can produce positive benefits. The IBCR value in this study is 1.09 , suggesting that ERAS is more cost-effective compared with conventional treatments.

Univariate sensitivity analyses were performed on three variables: bed turnover rate, hospitalisation cost and hospital profit margin. As the values of the three variables change, IBCR is still greater than 1 in most cases (Fig. 2). The univariate sensitivity analysis results indicated that the economics of ERAS protocols were relatively credible.

\section{Results of cost-minimisation analysis}

From the social perspective, the total cost per capita in ERAS group was $\$ 5958.67$, lower than $\$ 6725.80$ in the control group (Table 5). According to the principle of cost minimisation, ERAS is more economical and socially beneficial than conventional treatments.

The average daily cost per capita in the ERAS group was higher than that in the control group ( $\$ 669.51$ vs \$589.98). However, the total cost per capita (i.e. the area in Fig. 3) was lower in the ERAS group than in the control group due to a significant reduction in the average length of hospital stay ( $\$ 5958.67$ vs $\$ 6725.80$ ).

\section{Comprehensive analysis of cost changes}

The capital flow diagram for cost variation analysis are illustrated in Fig. 4. ERAS increases daily hospital expenses for patients, but reduces overall direct costs and productivity loss, resulting in a lower economic burden $(-\$ 1535.00)$. At the hospital level, there are two lines: on the one hand, the operating cost increases; on the other hand, the total medical income increases, and the final net income increases $(+\$ 3588.60)$. Thus, ERAS' total cost to society is decreased $(-\$ 767.13)$.

\section{Discussion \\ ERAS reduces the economic burden on patients}

Our study found that ERAS significantly decreased the economic burden of patients. Patients experienced a $\$ 1535.00$ cost reduction after ERAS implementation. Previous studies have shown that ERAS effectively saved \$1367.51 for each person [25]. Nelson et al's economic evaluation of colorectal surgery found that the net cost savings attributable to ERAS ranged from $\$ 2806.00$ to $\$ 5898.00$ [26]. Despite the cost savings of ERAS protocols vary in different application scenarios, they all prove that ERAS is a cost-effective intervention. The findings also show that the notable contribution to cost reduction was in direct medical costs. However, the study did not report the detailed composition of medical costs. Previous studies have noted that ERAS causes significant reductions in the cost of medication and disposable consumables [27, 28]. 
Table 2 Specific parameter values (\$)

\begin{tabular}{|c|c|c|c|c|c|}
\hline \multirow[t]{2}{*}{ Perspective } & \multirow[t]{2}{*}{ Content } & & \multicolumn{2}{|l|}{ Base value } & \multirow[t]{2}{*}{ Source } \\
\hline & & & Control group & ERAS group & \\
\hline \multirow[t]{12}{*}{ Patient } & Cost & Direct medical cost & & & \\
\hline & & Hospitalisation cost & $\begin{array}{l}9360.56 \\
(4854.36,16,483.53)\end{array}$ & $\begin{array}{l}7969.57 \\
(5310.19,10,754.38)\end{array}$ & Jing X [7] (2018) \\
\hline & & Direct non-medical cost & & & \\
\hline & & Food expenses & 824.12 & 742.68 & Wang D [6] (2019) \\
\hline & & Transportation & $(543.00,1105.24)$ & $(385.68,1099.67)$ & \\
\hline & & Employment of care workers & & & \\
\hline & & Others (accommodation, etc) & & & \\
\hline & & Indirect cost ${ }^{a}$ & & & \\
\hline & & Average hospital stays (d) & $\begin{array}{l}11.4 \\
(8.2,14.6)\end{array}$ & $\begin{array}{l}8.9 \\
(6.1,11.7)\end{array}$ & Chen L, et al [21] (2019) \\
\hline & & GDP per capita & $9134.20 / 365=25.03$ & $9134.20 / 365=25.03$ & China Statistical Yearbook [22] (2019) \\
\hline & Effect & Safety & & & \\
\hline & & Complication rate (\%) & $15 / 121 \times 100=12.4$ & $5 / 79 \times 100=6.33$ & Jing X [7] (2018) \\
\hline \multirow[t]{8}{*}{ Hospital } & Cost & Standard input cost ${ }^{b}$ & $\begin{array}{l}9360.56 \times 60 \%=5616.34 \\
(2912.62,9890.12)\end{array}$ & $\begin{array}{l}7969.57 \times 60 \%=4781.74 \\
(3186.11,6452.63)\end{array}$ & Jing X [7] (2018) \\
\hline & & Specific input cost ${ }^{c}$ & & & \\
\hline & & Salary of ERAS full-time nurse & - & 163.84 & Joliat GR, et al [10] (2016) \\
\hline & & Quarterly ERAS meeting cost & - & 1.34 & \\
\hline & & ERAS database cost & - & 44.52 & \\
\hline & & ERAS patient log cost & - & 1.78 & \\
\hline & Benefit & Hospital charges & $\begin{array}{l}9360.56 \\
(4854.36,16,483.53)\end{array}$ & $\begin{array}{l}7969.57 \\
(5310.19,10,754.38)\end{array}$ & Jing X [7] (2018) \\
\hline & & Number of patients admitted ${ }^{d}$ & $20 \times 30 \div 11.4$ & $20 \times 30 \div 8.9$ & Chen L, et al [21] (2019) \\
\hline \multirow[t]{10}{*}{ Society } & Cost & Standard input cost ${ }^{b}$ & $\begin{array}{l}9360.56 \times 60 \%=5616.34 \\
(2912.62,9890.12)\end{array}$ & $\begin{array}{l}7969.57 \times 60 \%=4781.74 \\
(3186.11,6452.63)\end{array}$ & Jing X [7] (2018) \\
\hline & & Specific input cost ${ }^{c}$ & & & \\
\hline & & Salary of ERAS full-time nurse & - & 163.84 & Joliat GR, et al [10] (2016) \\
\hline & & Quarterly ERAS meeting cost & - & 1.34 & \\
\hline & & ERAS database cost & - & 44.52 & \\
\hline & & ERAS patient log cost & - & 1.78 & \\
\hline & & Direct non-medical cost & $\begin{array}{l}824.12 \\
(543.00,1105.24)\end{array}$ & $\begin{array}{l}742.68 \\
(385.68,1099.67)\end{array}$ & Wang D [6] (2019) \\
\hline & & Indirect cost $^{a}$ & & & \\
\hline & & Average hospital stays (d) & $\begin{array}{l}11.4 \\
(8.2,14.6)\end{array}$ & $\begin{array}{l}8.9 \\
(6.1,11.7)\end{array}$ & Chen L, et al [21] (2019) \\
\hline & & GDP per capita & $9134.20 / 365=25.03$ & $9134.20 / 365=25.03$ & China Statistical Yearbook [22] (2019) \\
\hline
\end{tabular}

a Patient's indirect cost was calculated by multiplying the average length of stay by 2018 GDP per capita and dividing by 365

b Hospital's standard input (net cost) was approved by $60 \%$ of hospital charges [23]

c The specific input of ERAS was converted into the purchasing power parity of 100 yuan $=40.47$ Swiss francs [24]

${ }^{d}$ The absolute turnover of beds was assumed in this study because of the shortage of beds in tertiary hospitals (the average utilisation rate of beds in tertiary hospitals reached $97.5 \%$ in 2018 [18])

Assuming that there are 30 days per month and the department has 20 beds [18]. The formula for calculating hospital benefit was as follows: hospital benefit $=$ hospital charges per capita $\times$ number of patients admitted within one month $=$ average hospitalisation cost $\times(20 \times 30 \div$ average hospital stays $)$.

Table 3 Cost-effectiveness analysis results

\begin{tabular}{lll}
\hline Group & Cost (\$) & Effect (\%) \\
\hline Control group & $10,470.02$ & 12.40 \\
ERAS group & 8935.02 & 6.33 \\
\hline
\end{tabular}

\section{ERAS brings economic benefits to hospitals}

In this study, the hospital input costs increased by $\$ 41,024.73$ and benefits increased by $\$ 44,613.33$, leading to a profit increase of $\$ 3588.60$ for the hospital compared with the pre-ERAS. The economic assessment of 
Table 4 Cost-benefit analysis results

\begin{tabular}{llllll}
\hline Group & Cost (\$) & Benefit (\$) & Incremental cost (\$) & Incremental benefit (\$) & $\begin{array}{l}\text { IBCR } \\
(\Delta B / \Delta C)\end{array}$ \\
\hline Control group & $295,596.84$ & $492,661.05$ & $41,024.73$ & $44,613.33$ \\
ERAS group & $336,621.57$ & $537,274.38$ & & & 1.09 \\
\hline
\end{tabular}

\begin{tabular}{|c|c|c|c|c|}
\hline \multicolumn{5}{|l|}{ Variables } \\
\hline Hospital profit margin & 0.93 & & & 1.30 \\
\hline Hospitalization cost & 0.97 & & 1.17 & \\
\hline Bed turnover rate & & 1.09 & 1.18 & \\
\hline g.2 Results of univariate sensitivity ana & do analysis) & & 1.20 & 1.40 \\
\hline
\end{tabular}

Table 5 Cost-minimisation analysis results

\begin{tabular}{llll}
\hline Group & Overall cost (\$) & $\begin{array}{l}\text { Average } \\
\text { hospital stays } \\
\text { (d) }\end{array}$ & $\begin{array}{l}\text { Average } \\
\text { daily cost } \\
\mathbf{( \$ )}\end{array}$ \\
\hline Control group & 6725.80 & 11.4 & 589.98 \\
ERAS group & 5958.67 & 8.9 & 669.51 \\
\hline
\end{tabular}

Alberta's ERAS plan by Nguyen et al. reported that ERAS can bring in a return of $\$ 3.8$ for every $\$ 1$ invested [29]. The rate of return on investment of our study (1:1.09) was lower than that of the above study (1:3.8) [29], which may be related to the late start of ERAS and the fact that medical institutions have not yet reached the optimal state of coordination and unity in various aspects. ERAS implementation costs mainly include the cost of multidisciplinary team management and training and the cost of full-time nurses [30]. However, the decrease in the input of drugs and consumables is much higher than the salary and management expenditure. As a New Zealand study reported, the cost savings due to reduced postoperative resource utilization more than offset the input costs of ERAS [31]. This means that the value of technical labor is not well reflected under the current hospital fee compensation mechanism $[6,32]$.

\section{ERAS obtains social economic benefits}

Our results show a $\$ 767.13$ reduction in social cost per patient receiving ERAS. A study by Lee et al. pointed out that patients managed by ERAS had less productivity loss and lower readmission rates, which contributed to the reduction in overall social cost [33]. Readmission and follow-up costs after discharge reflect the long-term economic benefits of ERAS. Richardson et al. included readmission costs in their study and found that total costs in the ERAS group were still lower than in the conventional group [34].

\section{Mechanism by which ERAS works is to reduce average hospital stays and increase bed turnover rate}

Through the analysis of the cost variation, the economic benefits of ERAS are frequently attributed to the average length of hospital stay. The difference in average length of stay before and after ERAS translates into the cost difference, rather than the absolute length of hospital stay. The shortening of the average length of stay essentially reduces the number of ineffective hospitalisation days that are of no value to the patient and of little benefit to the hospital [35, 36]. Consequently, the consumption of social resources and the economic payment for patients are reduced. The essence of cost variation lies in the reduction of patients' productivity loss and the 


\section{Average length of stay (d)}

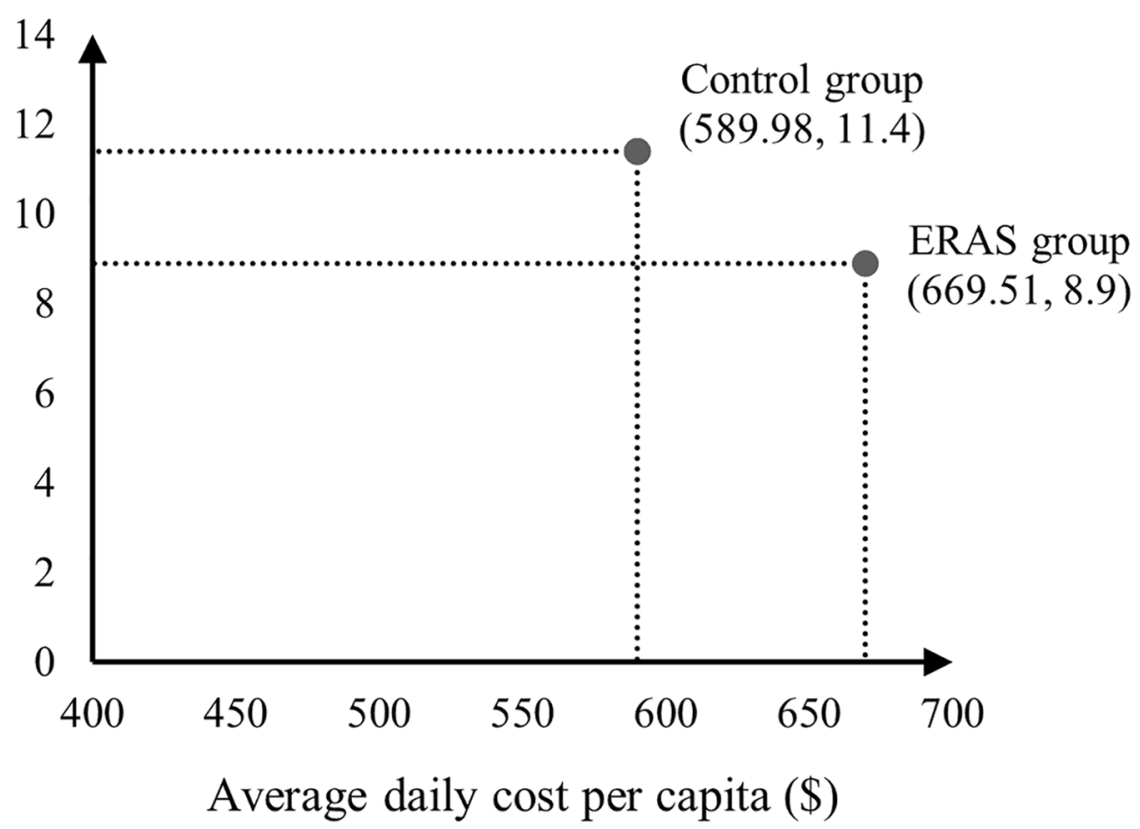

Fig. 3 Analysis of changes in total social costs of ERAS group and control group

consumption of ineffective services, but it increases the average daily hospitalisation cost.

Morever, the implementation of economic benefits of ERAS depends on the increase in the hospital bed turnover rate. Studies suggested that increased productivity in the health delivery system is expected to produce shorter hospital stays and lower costs [34]. Our research fully confirmed this finding. From the perspective of hospitals, the reduction in average length of stay increases bed turnover, especially in large tertiary hospitals, which are able to treat additional patients and generate more revenue with limited bed resources [37, 38]. From the perspective of society, the cost is actually transferred between patients and hospitals, and the ERAS input cost is offset by the benefits brought by the increase in bed turnover, ultimately achieving the overall reduction in consumption.

\section{Limitations}

There are three limitations to be acknowledged. Firstly, the establishment of cost structure has a decisive impact on the result of economic evaluation, and the difference in cost framework may lead to differences in results. Secondly, a cost difference may be driven by changes in causes and conditions of hepatectomy. Here, we ignored the interference of disease severity and surgical methods on the outcome of the disease and economic impact. Finally, this study only focused on the short-term economic value of ERAS.

\section{Implications}

ERAS offers a way to improve the efficiency of health resource use, so promoting overall patient recovery and reducing the average length of hospital stay should be a management priority in the future. Economic support is also supposed to be developed to hospitals.

Our study confirmed the economic benefits of ERAS using hepatectomy as an example, and provided an evaluation framework for the economic evaluation of ERAS. ERAS is also available in other scenarios, such as gastroenterology, gynecology, orthopedic, etc. ERAS may differ cost-effectively in different other scenarios, which will be further investigated in future studies. Similarly, to study the economic value of ERAS in other countries or regions, variations in parameters need to be considered to better match the actual situation.

\section{Conclusion}

In conclusion, the implementation of ERAS in hepatectomy reduces the direct and indirect economic burden on patients and society, increases hospital input costs 


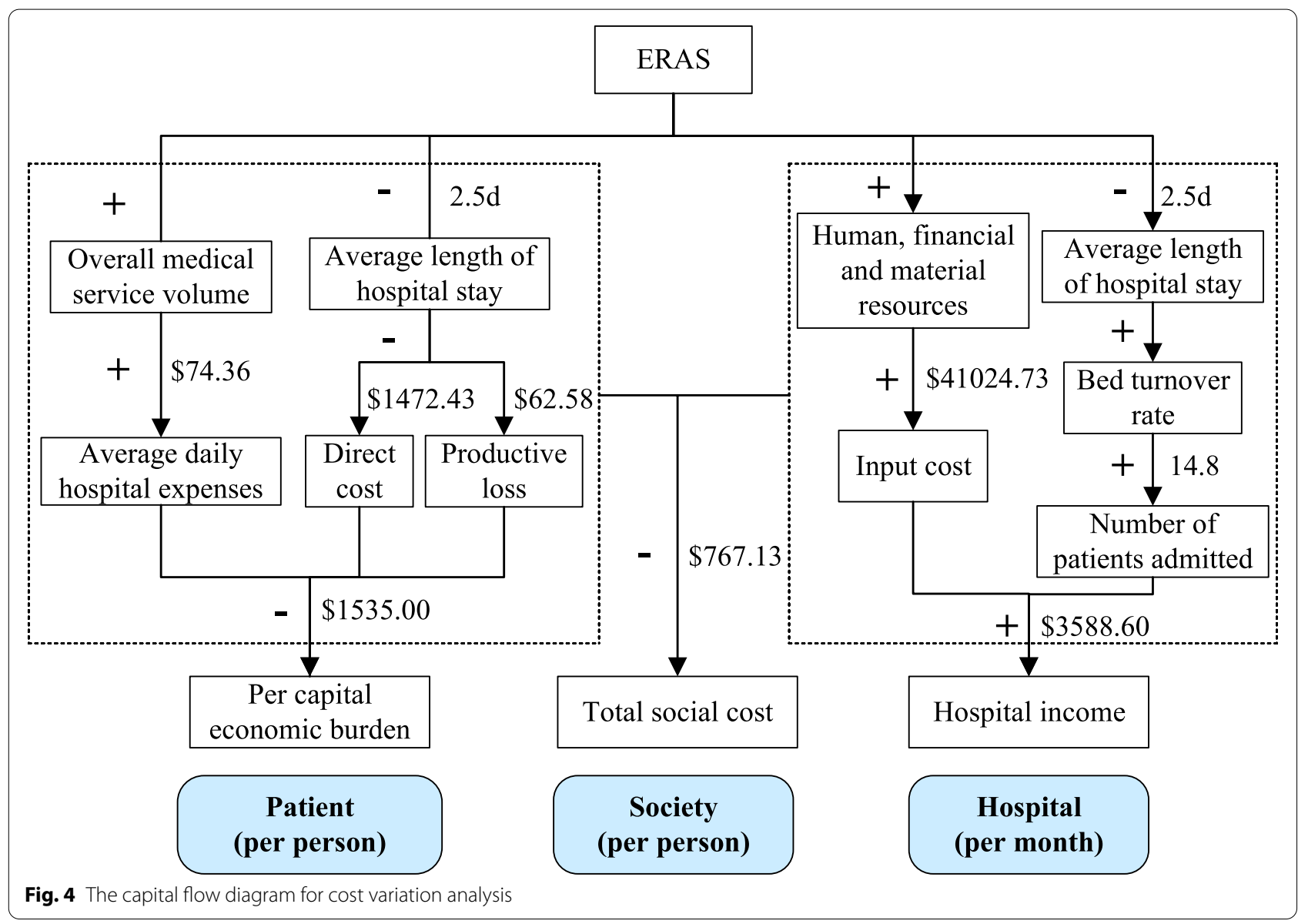

and promotes the efficiency of bed turnover. Shortening the average length of stay and improving bed turnover are the core issues in the mechanism to realise the economic benefits of ERAS.

\section{Abbreviations}

ERAS: Enhanced recovery after surgery; IBCR: Incremental benefit-cost ratio; FTS: Fast-track surgery; CPI: Consumer price index; CNY: Chinese yuan renminbi; USD: US dollar; CEA: Cost-effectiveness analysis; CBA: Cost-benefit analysis; CMA: Cost-minimisation analysis.

\section{Acknowledgements}

Not applicable.

\section{Authors' contributions}

All the authors have read the final draft and have provided comments and feedback. Write up: YD. The research topic and search strategies were designed and managed by all authors. YD and YZ developed the economic theoretical model and determined the basic parameters. YD worked with CJ to conduct economic evaluation. YZ reviewed papers. The authors read and approved the final manuscript.

\section{Funding}

This research was funded by the National Natural Science Foundation of China, Grant Number 71974046.
Availability of data and materials

Publicly available datasets were analysed in this study. This data can be found through the search strategy.

\section{Declarations}

Ethics approval and consent to participate

Not applicable.

\section{Consent for publication}

Not applicable.

strategy.

\section{Competing interests}

The authors declare that they have no competing interests.

\section{Author details}

${ }^{1}$ School of Medicine and Health Management, Tongji Medical College, Huazhong University of Science and Technology, No.13 Hangkong Road, Qiaokou District, Wuhan 430030, Hubei, China. ${ }^{2}$ Research Centre for Rural Health Service, Key Research Institute of Humanities \& Social Sciences of Hubei Provincial Department of Education, Wuhan 430030, China. ${ }^{3}$ Department of Surgery, Tongji Hospital, Tongji Medical College, Huazhong University of Science and Technology, Wuhan 430030, China.

Received: 25 May 2021 Accepted: 30 October 2021

Published online: 13 November 2021 


\section{References}

1. Chinese Medical Association Surgery Branch, Chinese Medical Association Anesthesiology Branch. Chinese expert consensus and path management guidelines for enhanced recovery after surgery (2018). Chin J Pract Surg. 2018;38(1):1-20.

2. Jeong $\mathrm{O}$, Kim HG. Implementation of enhanced recovery after surgery (ERAS) program in perioperative Management of Gastric Cancer Surgery: a Nationwide survey in Korea. J Gastric Cancer. 2019;19(1):72-82.

3. Liu C, Qiao Q, Luo M, Wu W, Lu G. Application research progress on enhanced recovery after surgery. Chin Nurs Res. 2019;33(2):261-4.

4. Zhang H. ERAS development helps "healthy China tour". China Hospital CEO. 2019;8:30-1.

5. Li P, Song W, Cong W. The history of enhanced recovery after surgery. Electron J Gen Stomatol. 2019:6(28):153.

6. Wang D. Health economic evaluation of "non-tube no fasting" enhanced recovery after minimally invasive esophagectomy: Zhengzhou University; 2019.

7. Jing X. Health economics evaluation research about enhanced recovery after surgery for clinical surgery: Qingdao University; 2018.

8. Yu L, Li H. Efficacy and cost-effectiveness of accelerated rehabilitation in the prevention of infection during perioperative period of aseptic surgery under pharmaceutical service. China Modern Doctor. 2016:54(29):108-10.

9. He S. Health economics analysis of the quality of life and prognosis of 36 patients with perioperative laparoscopic hepatectomy under the concept of enhanced recovery after surgery. For all Health (Late version). 2016;10(10):88-9

10. Joliat GR, Labgaa I, Hubner M, Blanc C, Griesser AC, Schafer M, et al. Costbenefit analysis of the implementation of an enhanced recovery program in liver surgery. World J Surg. 2016;40(10):2441-50.

11. Su Y, Zhang L, Ren L, Wei H, Zhen H, Wang F, et al. Meta-analysis of using enhanced recovery after surgery in open hepatectomy for liver cancer. Chin J Gen Surg. 2019;28(2):195-205.

12. Ren $Q$, Luo Y, Xiao F. Application of enhanced recovery after surgery in liver resection during perioperative period. Chin J Bases Clini Gen Surg. 2017:24(5):599-605.

13. Song W, Zou S. Application of enhanced recovery after surgery in setting of liver surgery: a Meta-analysis. Chin J Gen Surg. 2016;25(1):115-25.

14. Simpson JC, Moonesinghe SR, Grocott MP, Kuper M, McMeeking A, Oliver CM, et al. National enhanced recovery partnership advisory board. enhanced recovery from surgery in the UK: an audit of the enhanced recovery partnership programme 2009-2012. Br J Anaesth. 2015;115(4):560-8.

15. Cao Q, Ding Y, Wang B, Chen X. Clinical application of enhanced recovery after surgery (ERAS) program in laparoscopic hepatectomy for hepatolithiasis. Fu Bu Wai Ke. 2018;31(5):332-6.

16. Chen $H$, Zhang $D$, Lang $R$. Research progress of enhanced recovery after surgery in the perioperative period of liver resection. In: 2015 Beijing Annual Conference on Parenteral and Enteral Nutrition. Beijing: Beijing Medical Association; 2015. p. 131-6.

17. Larg A, Moss JR. Cost-of-illness studies: a guide to critical evaluation. Pharmacoeconomics. 2011;29(8):653-71

18. National Health Commission. China Health Statistics Yearbook. 2019

19. National Bureau of Statistics. China Statistical Yearbook 2017.

20. National Bureau of Statistics. China Statistical Yearbook. 2018

21. Chen $L$, Zhang $H$, Lin $Y$. The application value of enhanced recovery after surgery concept in the nursing of laparoscopic partial hepatectomy. Chin Manipul Rehabil Med. 2019;10(6):74-6.

22. National Bureau of Statistics. China Statistical Yearbook. 2019.
23. Xue LL, Zhang JZ, Shen HX, Hou Y, Ai L, Cui XM. The application of rapid rehabilitation model of multidisciplinary cooperation in cesarean section and the evaluation of health economics. Zhonghua Yi Xue Za Zhi. 2019;99(42):3335-9

24. Hexun. Just now, RMB broke 7! 100 yuan in the United States immediately becomes 52 yuan, what is the global purchasing power of the yuan? 2019 [cited 2021-05-16]. https://forex.hexun.com/2019-08-06/ 198106361.html.

25. Jing X, Zhang B, Xing S, Tian L, Wang X, Zhou M, et al. Cost-benefit analysis of enhanced recovery after hepatectomy in Chinese Han population. Medicine (Baltimore). 2018 Aug;97(34):e11957.

26. Nelson G, Kiyang LN, Crumley ET, Chuck A, Nguyen T, Faris P, et al. Implementation of enhanced recovery after surgery (ERAS) across a provincial healthcare system: the ERAS Alberta colorectal surgery experience. World J Surg. 2016;40(5):1092-103.

27. Song $X, M a$ J. Anesthesia management under the guidance of ERAS concept. J Shandong Univ (Health Sci). 2019;57(9):12-7.

28. Moningi S, Patki A, Padhy N, Ramachandran G. Enhanced recovery after surgery: an anesthesiologist's perspective. J Anaesthesiol Clin Pharmacol. 2019:35(Suppl 1):S5-S13.

29. Thanh NX, Chuck AW, Wasylak T, Lawrence J, Faris P, Ljungqvist O, et al. An economic evaluation of the enhanced recovery after surgery (ERAS) multisite implementation program for colorectal surgery in Alberta. Can $J$ Surg. 2016;59(6):415-21.

30. Roulin D, Donadini A, Gander S, Griesser AC, Blanc C, Hübner M, et al. Cost-effectiveness of the implementation of an enhanced recovery protocol for colorectal surgery. Br J Surg. 2013;100(8):1108-14.

31. Sammour T, Zargar-Shoshtari K, Bhat A, Kahokehr A, Hill AG. A programme of enhanced recovery after surgery (ERAS) is a cost-effective intervention in elective colonic surgery. N Z Med J. 2010;123(1319):61-70.

32. Ye B. Study on hospitalisation expenses and its influential factors among esophageal Cancer patients receiving surgeries: Zhejiang University; 2015.

33. Lee L, Mata J, Ghitulescu GA, Boutros M, Charlebois P, Stein B, Liberman $A S$, et al. Cost-effectiveness of enhanced recovery versus conventional perioperative management for colorectal surgery. Ann Surg 2015;262(6):1026-1033

34. Richardson J, Di Fabio F, Clarke H, Bajalan M, Davids J, Abu HM. Implementation of enhanced recovery programme for laparoscopic distal pancreatectomy: feasibility, safety and cost analysis. Pancreatology. 2015;15(2):185-90.

35. Li G. Research on influencing factors analysis and management strategies to ineffective hospitalisation days of surgical patients in three-level general hospitals: Huazhong University of Science and Technology; 2017.

36. Rodríguez FC, Morón Ide L, Martínez AD, Rodríguez LL, Lucena FM, Fernández MT. Appropriateness of hospital stays in a pulmonology department. Arch Bronconeumol. 2007:43(8):439-44.

37. He G, Hong H, Cheng Y, Jiang Z, Zhou C, Liu H, et al. Application of the concept of enhanced recovery after surgery in laparoscopic hepatectomy. Chin J Gen Surg. 2018;33(8):635-7.

38. Kim JW, Kim WS, Cheong J-H, Hyung WJ, Choi S-H, Noh SH. Safety and efficacy of fast-track surgery in laparoscopic distal gastrectomy for gastric cancer: a randomized clinical trial. World J Surg. 2012;33(8):635-7.

\section{Publisher's Note}

Springer Nature remains neutral with regard to jurisdictional claims in published maps and institutional affiliations. 